\title{
FINANZAS Y SALUD MENTAL
}

\author{
Fernando López \\ Profesor de Finanzas, Universidad Alberto Hurtado
}

\begin{abstract}
Resumen
Los esfuerzos para cuantificar y mitigar los daños asociados a eventos inesperados que producen perjuicios económicos, como desastres naturales o caídas inesperadas en el valor de los activos, se concentran en los daños materiales. Sin embargo, esta aproximación ignora un tipo de daño altamente relevante para las personas afectadas y la sociedad: el daño a la salud mental. Este artículo discute la medida en que los servicios financieros podrían contribuir a mitigar el efecto de shocks adversos en la salud mental, presenta una selección de la evidencia científica disponible y describe los desafíos que enfrentan los estudios orientados a analizar esta hipótesis.
\end{abstract}

\section{Shocks adversos afectan la salud mental}

El 16 de Septiembre de 2015, Chile fue golpeado por un terremoto de magnitud 8,4 en la escala de Richter. De acuerdo a información oficial de la Oficina Nacional de Emergencia (ONEMI), se registraron 15 personas fallecidas, 28 mil personas damnificadas, 2.400 viviendas destruidas y 2.700 viviendas no habitables. Tal como ocurre en la mayoría de los países que experimentan algún desastre natural, u otro tipo de evento adverso que produce perjuicios económicos, las mediciones oficiales se enfocan en cuantificar y mitigar los daños materiales asociados al evento. Sin embargo, estas mediciones ignoran el efecto de estos daños en la salud mental de las personas.

Una serie de estudios recientes muestran que los shocks que producen daños materiales pueden tener efectos significativos en la salud mental de las personas. Por ejemplo, caídas abruptas en los precios accionarios en el estado de California (EEUU) en el periodo 1983-2011, estuvieron fuertemente relacionadas con aumentos en el número de admisiones hospitalarias por condiciones como ansiedad, crisis de pánico y depresión (Engelberg y Parsons, forthcoming). Por su parte, una reducción repentina en los beneficios de los planes de pensión en Holanda en el año 2006 estuvo acompañada por un aumento significativo en las tasas de depresión de los pensionados afectados por dicha reforma (De Grip, Lindeboom y Montizaan, 2012). Otros estudios incluso han mostrado que aumentos en el desempleo en Estados Unidos (Ruhm, 2000) y la crisis inmobiliaria reciente coincidieron con aumentos en las tasas de suicidio en Grecia (Economou et al., 2013) y Hong Kong (Lee et al., 2010).
Importancia de estudiar la relación entre servicios financieros y salud mental

Existen al menos tres razones por las cuales es importante estudiar si el uso de servicios financieros ayuda a mitigar el efecto de shocks adversos en la salud mental. Primero, si definimos el bienestar individual como la frecuencia e intensidad de sensaciones de agrado y desagrado, una definición utilizada por el psicólogo y premio nobel de economía, Daniel Kahneman, junto a sus coautores (Kahneman, Wakker y Sarin, 1997), la salud mental es un determinante clave del bienestar individual. En efecto, un deterioro de la salud mental puede ser altamente debilitante porque afecta el estado de ánimo, la capacidad de descanso y, en general, la capacidad de funcionamiento de las personas. Del mismo modo, además de ser una de las condiciones más caras para el sistema de salud de economías desarrolladas como Estados Unidos (Soni, 2009), algunos estudios sugieren que las condiciones de salud mental reducen la productividad (Stewart et al., 2003; Kessler et al. 2006; Beck et al. 2011) y la capacidad de generar ingresos (Kessler et al., 2008; Helliwell, Layard y Sachs, 2013).

Segundo, los mercados financieros ofrecen una serie de mecanismos que contribuyen a mitigar las pérdidas asociadas a shocks que producen perjuicios económicos. Por ejemplo, los seguros entregan una compensación directa a las personas que sufren un siniestro cubierto por la póliza. Por su parte, el ahorro y el acceso a crédito mejoran la capacidad de las personas para enfrentar de manera inmediata las consecuencias negativas de un shock en comparación a aquellas que no tienen ahorros o 
acceso a crédito. Sin embargo, a la fecha existen pocos estudios que permitan cuantificar la medida en que el uso de estos servicios financieros contribuye a reducir la probabilidad de que se deteriore la salud mental de las personas afectadas por un shock adverso.

Tercero, a pesar de su disponibilidad tanto en Chile como en el mundo, pocas personas cuentan con productos financieros que permiten mitigar las consecuencias de eventos adversos. De acuerdo a estimaciones del Banco Mundial (Cull, DemirgüçKunt, Morduch, 2012), solo la mitad de los habitantes del mundo tienen una cuenta con un intermediario financiero. Considerando el caso de los terremotos, a pesar de estar ubicados en áreas altamente sísmicas, menos de un 30\% de las viviendas chilenas (SVS, 2012) y menos de un $20 \%$ de las viviendas en el estado de California (Scism, 2015) se encuentran aseguradas contra terremotos. En este contexto, entender el efecto del uso de servicios financieros en la salud mental de personas afectadas por shocks económicos adversos puede ser informativo para políticas orientadas a promover un mayor acceso y uso de servicios financieros.

\section{Evidencia}

Existen pocos estudios que analizan la relación entre uso de servicios financieros y salud mental. Una excepción es un estudio reciente (López, 2015) que analiza la medida en que el uso de servicios financieros, como seguros y productos de ahorro, ayudaron a reducir los síntomas de Trastorno de Estrés PostTraumático (TEPT) asociado al terremoto que afectó a Chile en 2010'. El TEPT es un trastorno ansioso asociado a una limitación en la capacidad de reacción de las personas que se caracteriza por sensaciones de miedo y estrés cuando estas no están en riesgo ${ }^{2}$. Los resultados de este estudio sugieren que personas con seguro de terremoto tuvieron una menor probabilidad de desarrollar síntomas de TEPT en áreas más afectadas. Estimaciones conservadoras sugieren que la probabilidad de presentar síntomas de TEPT fue al menos un 50\% menor para las personas con seguro contra terremoto. Por el contrario, no se encontraron diferencias significativas en la probabilidad de desarrollar síntomas de estrés post-traumático para personas que tenían productos de ahorro o crédito.

Una serie de estudios relacionados analiza el efecto de los servicios financieros en el bienestar general de las personas. Sin embargo, estos consideran medidas de consumo (Townsend, 1994; Morduch, 1995; Gruber, 1997) y la habilidad de pagar cuentas (Morse, 2011; Melzer, 2011) como indicadores de bienestar. A pesar de que estos indicadores tienen la ventaja de ser fáciles de medir, en la práctica, las personas pueden reaccionar a eventos económicos adversos ajustando distintos tipos de gastos como consumo, pago de préstamos, inversiones en capital humano o salud. Por esta razón, es difícil establecer una relación entre uso de servicios financieros y bienestar a partir de indicadores de consumo y cumplimiento de compromisos financieros.

\section{Desafíos de esta investigación}

Estudiar la relación entre el uso de servicios financieros y la salud mental presenta desafíos complejos para los investigadores. Primero, existen pocas bases de datos que tengan información detallada de salud mental y uso de servicios financieros a nivel individual. En efecto, las fuentes de datos con información de servicios financieros están diseñadas por economistas y sociólogos que tienen por objeto dimensionar la participación de las personas en el mercado financiero. Por su parte, los instrumentos diseñados para medir la salud mental son construidos por psicólogos y psiquiatras, principalmente con fines médicos y epidemiológicos. Sin bases de datos que tengan información de servicios financieros y salud mental, no es posible caracterizar su relación.

Segundo, el diseño metodológico de los estudios debe contemplar estrategias que permitan aislar el efecto de los servicios financieros en la salud mental de las personas de otros factores que también podrían determinar tanto el uso de servicios financieros como la salud mental. Para ilustrar este punto, consideremos el caso de personas afectadas por un terremoto. Las personas con mejores condiciones socioeconómicas tendrán una mayor probabilidad de tener seguro contra terremoto $y$, al mismo tiempo, más recursos para hacer frente a los daños materiales causados por un eventual terremoto. En este contexto, si estas personas tienen una menor probabilidad de desarrollar condiciones de salud mental, es difícil determinar si esta relación se debe a que tienen seguro contra terremoto o a que tienen mejores condiciones socioeconómicas para hacer frente a estos eventos.

(1) Este terremoto se encuentra entre los 10 más intensos registrados desde 1900 , afectó a un $80 \%$ de la población y produjo daños materiales por cerca de US\$30 mil millones ( $17 \%$ del PIB).

(2) Algunos síntomas del TEPT son: re-experimentación del trauma a través de pensamientos o pesadillas, elusión de situaciones o estímulos relacionados con el evento traumático, pérdida de la reactividad general y síntomas de hiperalerta como enojo o dificultad para dormir. 


\section{Conclusión}

Las condiciones de salud mental son costosas para las personas y la sociedad. En la medida que shocks económicos adversos tengan la capacidad de dañar la salud mental de las personas, es importante analizar si el uso de los servicios financieros contribuye a mitigar esta relación. Esta tarea presenta una serie de desafíos metodológicos que ciertamente se beneficiarían de un trabajo multidisciplinario que integre académicos en economía y finanzas con expertos del área de la salud mental.

\section{Referencias}

Baird, Sarah, de Hoop, Jacobus and Berk Özler, 2013, Income Shocks and Adolescent Mental Health, Journal of Human Resources, 48 (2), 370-403.

Beck, Arne, Crain, A. Lauren, Solberg, Leif I., Unützer, Jürgen, Glasgow, Russell E., Maciosek, Michael V. and Robin Whitebird, 2011, Severity of Depression and Magnitude of Productivity Loss, Annals of Family Medicine, 9 (4), 305-311.

Cull, Robert, Demirgüç-Kunt, Asli and Jonathan Morduch, 2012, Banking the World: Empirical Foundations of Financial Inclusion, MIT University Press.

De Grip, Andries, Lindeboom, Maarten and Raymond Montizaan, 2012, Shattered Dreams: The Effects of Changing the Pension System Late in the Game, The Economic Journal, 122 (599), 1-25.

Economou, M., Madianos, M., Peppou, L. E., Patelakis, A., \& Stefanis, C. N., 2013, Major depression in the Era of economic crisis: A replication of a cross-sectional study across Greece. Journal of Affective Disorders, 145(3), 308-314.

Engelberg and Parsons, Forthcoming, Worrying about the stock market: Evidence from hospital admissions, Journal of Finance.

Gruber, Jonathan, 1997, The consumption smoothing benefits of unemployment insurance, American Economic Review, 87 (1): 192-205.

Helliwell, John, Richard Layard and Jeffrey Sachs, 2013, World happiness report, The Earth Institute - Columbia University.

Kahneman, Daniel, Peter Wakker and Rakesh Sarin, 1997, Back to Bentham? Explorations of experienced utility, Quarterly Journal of Economics, 112 (2), 375-406.

Kessler, Ronald C., Akiskal, Hagop S., Ames, Minnie, Birnbaum, Howard, Greenberg, Paul, Hirschfeld, Robert M., Jin, Robert, Merikangas, Kathleen R., Simon, Gregory E. and Phillip S. Wang,
2006, Prevalence and Effects of Mood Disorders on Work Performance in a Nationally Representative Sample of U.S. Workers, American Journal of Psychiatry, 163 (9),1561-1568.

Kessler, Ronald C., Heeringa, Steven, Lakoma, Matthew D., Petukhova, Maria, Rupp, Agness E., Schoenbaum, Michael, Wang, Phillip S. and Adam M. Zaslavsky, 2008, Individual and Societal Effects of Mental Disorders on Earnings in the United States: Results From the National Comorbidity Survey Replication, American Journal of Psychiatry, 165 (6), 703-711.

Lee, S., Guo, W. J., Tsang, A., Mak, A. D. P., Wu, J., Ng, K. L., \& Kwok, K., 2010, Evidence for the 2008 economic crisis exacerbating depression in Hong Kong. Journal of Affective Disorders, 126(12), 125-133.

Lopez, Fernando, 2015, Financial services and individual welfare, working paper, Universidad Alberto Hurtado.

Melzer, Brian, 2011, "The real costs of credit access: Evidence from the payday lending market," Quarterly Journal of Economics, 126, 517-555.

Morduch, Jonathan, 1995, Income smoothing and consumption smoothing. Journal of Economic Perspectives, 9 (3), 103-114.

Morse, Adair, 2011, Payday Lenders: Heroes or Villains?, Journal of Financial Economics, 102 (1), 28-44.

Ruhm, Christopher, 2000, Are recessions good for your health?, Quarterly Journal of Economics, 115 (2), 617-650.

Scism, Leslie, 30 de Agosto de 2015, California's Earthquake Problem: People Aren't Scared Enough About Them, obtenido de Wall Street Journal http://www.wsj.com/articles/california-pushes-homeowners-to-insure-against-earthquakes-1440980138

Soni, Anita, 2009, The five most costly conditions, 1996 and 2006: Estimates for the U.S. civilian non-institutionalized population, Agency for Healthcare Research and Quality, Statistical Brief 248.

Superintendencia de Valores y Seguros de Chile (SVS), 2012, Terremoto 2010, Análisis e impacto del 27-F en el mercado asegurador, Superintendencia de Valores y Seguros, Santiago.

Stewart, Walter F., Ricci, Judith A., Chee, Elsbeth, Hahn, Steven R. and David Morganstein, 2003, Cost of lost productive work time among US workers with depression, Journal of American Medical Association; 289 (23), 3135-3144.

Townsend, Robert, 1994, Risk and insurance in village India, Econometrica, 62 (3), 539-591. 